Research Article

\title{
Microbial Predominance and Antimicrobial Resistance in a Tertiary Hospital in Northwest China: A Six-Year Retrospective Study of Outpatients and Patients Visiting the Emergency Department
}

\author{
Caifeng Wang $\mathbb{D}^{1},{ }^{1}$ Wen $\mathrm{Li}^{2}{ }^{2}$ Juanjuan Gao, ${ }^{3}$ Dancheng Zhang, ${ }^{4}$ Yali Li, ${ }^{2}$ Fang Li, \\ and Jine Lei $\mathbb{D}^{2}$ \\ ${ }^{1}$ Department of Emergency, First Affiliated Hospital, Xi'an Jiaotong University, Xi'an, Shaanxi Province, China \\ ${ }^{2}$ Department of Clinical Laboratory, First Affiliated Hospital, Xi'an Jiaotong University, Xi'an, Shaanxi Province, China \\ ${ }^{3}$ Biological Specimen Management, First Affiliated Hospital, Xi'an Jiaotong University, Xi'an, Shaanxi Province, China \\ ${ }^{4}$ College of Laboratory Medicine, Chongqing Medical University, Chongqing, China
}

Correspondence should be addressed to Caifeng Wang; wangcf33@mail.xjtu.edu.cn

Received 16 September 2020; Accepted 28 October 2020; Published 29 November 2020

Academic Editor: Pietro Mastroeni

Copyright (C) 2020 Caifeng Wang et al. This is an open access article distributed under the Creative Commons Attribution License, which permits unrestricted use, distribution, and reproduction in any medium, provided the original work is properly cited.

Background. With the wide use of antibiotics, antimicrobial resistance becomes a serious issue. Timely understanding of microbial pathogen profiles and the change of antimicrobial resistance provide an important guidance for effective and optimized use of antibiotics in local healthcare systems. The aim was to investigate the characteristics of microbial species and their antimicrobial resistances in a tertiary hospital with an Emergency Department and outpatient clinics for a period of six years. Methodology. A retrospective study was conducted using the HIS database of a tertiary hospital between 2013 and 2018. Antimicrobial susceptibility was tested by automated systems and/or the Kirby-Bauer disc diffusion method. The data were analyzed using the WHONET 5.6 software. The Cochran-Armitage test was used to study the trends over the period of research. Results. In a total of 19,028 specimens submitted for microbial tests during the period from 49 units of the hospital, only the samples from the Emergency Department and Kidney Transplantation Clinic showed an annually significant increase $(P<0.001)$. More than 200 species with $46.4 \%$ gram-positive cocci and $45.3 \%$ gram-negative bacilli were identified in the 3,849 nonrepetitive isolates. The methicillin-resistant S. aureus and S. epidermidis rates were $25.1 \%$ and $74.6 \%$, respectively. $60.9 \%$ E. coli and $33.5 \%$ K. pneumonia samples carried extended-spectrum- $\beta$-lactamase. All Staphylococci and Enterococci samples were not resistant to linezolid, vancomycin, and tigecycline. In addition, only $0.01 \%$ E. coli, $1.1 \%$ K. pneumonia, and $18.7 \%$ P. aeruginosa isolates showed resistance to carbapenems. Conclusions. Vancomycin, linezolid and tigecycline were the most effective antibiotics for outpatients with gram-positive infection. Carbapenems were the most effective antibiotics for gram-negative infection. There was no significant annual increase of common multidrug resistances.

\section{Introduction}

Increased usage of broad-spectrum antimicrobial treatments leads to microbial resistance to the treatments that were originally sensitive. The increased antimicrobial resistance (AMR) limits treatment options and is considered as a global challenge to public health with increased morbidity, mortality, and healthcare costs [1] To accurately estimate the challenge, the surveillance of AMR profiling at local, regional, and national levels has been employed to understand the global trends on the type of predominant pathogens and their respective resistance profiling [2]. In China, the surveillance of AMR is established at the hospital, provincial, and national levels. For example, CHINET is one of well-established national surveillance networks for AMR [3-6]. This network 
TABle 1: Annual number and percentage (\%) of the specimens submitted from outpatient clinics and emergency department for the laboratory tests from 2013 to 2018 .

\begin{tabular}{|c|c|c|c|c|c|c|c|c|c|c|c|c|c|c|}
\hline \multirow{2}{*}{ Department } & \multicolumn{2}{|c|}{2013} & \multicolumn{2}{|c|}{2014} & \multicolumn{2}{|c|}{2015} & \multicolumn{2}{|c|}{2016} & \multicolumn{2}{|c|}{2017} & \multicolumn{2}{|c|}{2018} & \multirow{2}{*}{$P$ value } & \multirow{2}{*}{ Trend } \\
\hline & $N$ & $\%$ & $N$ & $\%$ & $N$ & $\%$ & $N$ & $\%$ & $N$ & $\%$ & $N$ & $\%$ & & \\
\hline Emergency & 469 & 20.4 & 490 & 19.7 & 517 & 21.0 & 1017 & 32.5 & 1046 & 32.0 & 2.306 & 42.9 & $<0.001$ & $\uparrow$ \\
\hline Urology & 400 & 17.4 & 710 & 28.5 & 497 & 20.1 & 426 & 13.6 & 673 & 20.6 & 891 & 16.6 & $<0.001$ & $\downarrow$ \\
\hline Nephrology & 559 & 24.3 & 427 & 17.2 & 491 & 19.9 & 511 & 16.3 & 466 & 14.3 & 581 & 10.8 & $<0.001$ & $\downarrow$ \\
\hline Respiratory & 272 & 11.8 & 211 & 8.5 & 381 & 15.4 & 491 & 15.7 & 300 & 9.2 & 475 & 8.8 & 0.002 & $\downarrow$ \\
\hline Convenient* & 120 & 5.2 & 129 & 5.2 & 134 & 5.4 & 126 & 4.0 & 115 & 3.5 & 160 & 3.0 & $<0.001$ & $\downarrow$ \\
\hline Rheumatology & 150 & 6.5 & 71 & 2.9 & 56 & 2.3 & 68 & 2.2 & 85 & 2.6 & 124 & 2.3 & $<0.001$ & $\downarrow$ \\
\hline Peritoneal dialysis & 45 & 2.0 & 91 & 3.7 & 82 & 3.3 & 64 & 2.0 & 69 & 2.1 & 115 & 2.1 & 0.083 & No change \\
\hline Kidney transplant & 33 & 1.4 & 52 & 2.1 & 25 & 1.0 & 36 & 1.2 & 69 & 2.1 & 143 & 2.7 & 0.0043 & $\uparrow$ \\
\hline Gynecology & 32 & 1.4 & 74 & 3.0 & 80 & 3.2 & 68 & 2.2 & 50 & 1.5 & 53 & 1.0 & $<0.001$ & $\downarrow$ \\
\hline Breast surgery & $* *$ & $* *$ & $* *$ & $* *$ & $* *$ & $* *$ & 62 & 2.0 & 103 & 3.2 & 140 & 2.6 & $* *$ & $* *$ \\
\hline Other ${ }^{* * *}$ & 223 & 9.7 & 232 & 9.3 & 204 & 8.3 & 258 & 8.3 & 290 & 8.9 & 390 & 7.3 & 0.012 & $\downarrow$ \\
\hline Total & 2.303 & 100.0 & 2.487 & 100.0 & 2.467 & 100.0 & 3.127 & 100.0 & 3.266 & 100.0 & 5.378 & 100.0 & & \\
\hline
\end{tabular}

${ }^{*}$ Convenient clinic helps to meet simple requests of the patients with frequent visits, such as to fill in the prescription and/or to obtain the regular laboratory tests. ${ }^{* *}$ Data not available. ${ }^{* * *}$ Other outpatient clinics include dermatology clinic, blood purification clinic, infectious disease clinic, hematology clinic, oncology clinic, pediatric clinic, Chinese medicine clinic, digestive medicine clinic, otolaryngology clinic, general surgery clinic, orthopedic surgery clinic, endocrinology clinic, ophthalmology clinic, thoracic surgery clinic, hepatobiliary surgery clinic, neurology clinic, obstetrics clinic, cardiovascular disease clinic, cardiac and vascular clinic, neurosurgery clinic, geriatric surgery clinic, oncology surgery clinic, Chinese medicine clinic (Xingshan campus), neonatal clinic, chest clinic, burn plastic surgery clinic, reproductive medicine clinic, cardiovascular surgery clinic, biotherapy consultation clinic, stomatology clinic, general medicine clinic, tumor radiotherapy clinic, structural heart disease clinic, famous senior Chinese medicine clinic, peripheral vascular clinic, rehabilitation clinic (Xingshan campus), Lugang obstetrics and gynecology clinic, pain clinic, and vascular surgery clinic.

recently reported that the frequency of gram-negative bacilli among clinical isolates was over twice higher than that of gram-positive cocci $[3,4]$. In addition, it is critical to recognize that China is a large country with variety of microbial occurrences and AMR profiling. For example, Xiao et al. reported that the percentage of MRSA (methicillin-resistant Staphylococcus aureus) in North Central and South Central China is higher than other regions [7]. The geographic variety is likely associated with regional differences in socioeconomic development [7]. Therefore, the microbial predominance and AMR profiling in a specific region and province is not always consistent with the trend at a national level. Indeed, the surveillance of AMR at the hospital and provincial levels is a valuable complement to the national surveillance. Furthermore, the updated AMR profiling at the hospital and regional levels is particularly important for physicians to determine the adequate empiric antimicrobial therapy in clinical practice. Therefore, the objectives of this study were to analyze the data from a six-year surveillance of AMR at an university affiliated tertiary hospital and to obtain the characteristics of microbial species and their antimicrobial resistances for both surveillance and clinical practice perspectives.

The routine surveillance was mainly focused on hospitalacquired infections, and most specimens were collected from inpatients with severe infections [8], while community-acquired infections from outpatient service were always underreported $[2,8]$. Therefore, in our study, we tried to bridge the gap and studied the microbial predominance and AMR profiling in outpatient clinics and emergency department in a specific region of Northwestern China. Our results were also compared with the findings from the CHINET.

\section{Materials and Methods}

2.1. Data Source. This study was performed at the First Affiliated Hospital of Xi' an Jiaotong University, which is one of the largest hospitals in northwest China. It provides medical and surgical care to the residents of Shaanxi Province with a total population 37.33 million. The institutional review board at the First Affiliated Hospital approved this study (No: XJTU1AF2017LSK-83) and written informed consent was not required because the laboratory tests for microbiology are part of standard care and the patient records were excluded prior to this analysis. Archived laboratory data between 2013 and 2018 were retrieved from the HIS database of the hospital for analysis. A total of 19,028 specimens were collected from the emergency department and 49 outpatient clinics. A full list of these clinics is provided in Table 1. The specimens included urine, blood, prostatic fluid, sputum, pleural effusion and ascites, stool, dialysate, pus, secretion, drainage, cerebrospinal fluid, and bronchoalveolar lavage fluid.

2.2. Isolate Identification. The clinical specimens were processed according to the recommended microbiological procedures as previously described [9-12]. Species were identified through colony morphology, conventional biochemical reactions and/or the use of an automated system (bioMerieux, Marcy l'Etoile, France).

2.3. Antibiotic Sensitivity. Mueller-Hinton agar (MH agar) and $\mathrm{MH}$ agar with 5\% sheep blood Haemophilus test medium (HTM) was purchased from bioMerieux, Marcy l'Etoile, France. The following ATCC strains were used as references: Escherichia coli (ATCC 25922), Pseudomonas 
TABLE 2: Annual number and percentage (\%) of the types of specimens submitted for the laboratory tests from outpatients and patients visiting emergency department between 2013 and 2018.

\begin{tabular}{lccccccccccccccc}
\hline \multirow{2}{*}{ Specimen type } & \multicolumn{2}{c}{2013} & \multicolumn{2}{c}{2014} & \multicolumn{2}{c}{2015} & \multicolumn{2}{c}{2016} & \multicolumn{2}{c}{2017} & \multicolumn{2}{c}{2018} & \multicolumn{2}{c}{$P$ value } & Trend \\
& $N$ & $\%$ & $N$ & $\%$ & $N$ & $\%$ & $N$ & $\%$ & $N$ & $\%$ & $N$ & $\%$ & \\
\hline Urine & 919 & 39.9 & 778 & 31.3 & 805 & 32.6 & 970 & 31.0 & 1.178 & 36.1 & 1.733 & 32.2 & 0.025 & $\downarrow$ \\
Sputum & 395 & 17.2 & 262 & 10.5 & 393 & 15.9 & 672 & 21.5 & 515 & 15.8 & 1.077 & 20.0 & $<0.001$ & $\uparrow$ \\
Whole blood & 231 & 10.0 & 284 & 11.4 & 276 & 11.2 & 511 & 16.3 & 636 & 19.5 & 1.201 & 22.3 & $<0.001$ & $\uparrow$ & \\
Prostatic fluid & 248 & 10.8 & 491 & 19.7 & 321 & 13.0 & 220 & 7.0 & 254 & 7.8 & 348 & 6.5 & $<0.001$ & $\downarrow$ \\
Pus* & 29 & 1.3 & 33 & 1.3 & 43 & 1.7 & 81 & 2.6 & 142 & 4.3 & 179 & 3.3 & $<0.001$ & $\uparrow$ \\
Dialysate & 29 & 1.3 & 85 & 3.4 & 76 & 3.1 & 74 & 2.4 & 96 & 2.9 & 147 & 2.7 & 0.235 & No change \\
Peritoneal drainage & 9 & 0.4 & 52 & 2.1 & 45 & 1.8 & 82 & 2.6 & 91 & 2.8 & 48 & 0.9 & 0.709 & No change \\
Pleural effusion and ascites & 8 & 0.3 & 25 & 1.0 & 17 & 0.7 & 52 & 1.7 & 31 & 0.9 & 55 & 1.0 & 0.117 & No change \\
Others** & 435 & 18.9 & 477 & 19.2 & 491 & 19.9 & 465 & 14.9 & 323 & 9.9 & 590 & 11.0 & $<0.001$ & $\downarrow$ \\
Total & 2.303 & 100.0 & 2.487 & 100.0 & 2.467 & 100.0 & 3.127 & 100.0 & 3.266 & 100.0 & 5.378 & 100.0 & & \\
\hline
\end{tabular}

* Pus was collected from skin and mammary gland. ${ }^{* *}$ Others mainly include cerebrospinal fluid, joint fluid, feces, reproductive tract secretions and bone marrow.

aeruginosa (ATCC 27853), Staphylococcus aureus (ATCC 25923), and Enterococcus faecallis (ATCC 29212). An automated system (bioMerieux, Marcy l'Etoile, France) and/or the Kirby-Bauer Disc Diffusion Method were used to test for antimicrobial susceptibility according to the guidelines of the Clinical Laboratory Standards Institute. The sensitivity breakpoint of cefoperazone/sulbactam referred to that of cefoperazone.

The following antibiotics were tested against available isolates: piperacillin, oxacillin, ampicillin, ampicillin/sulbactam, piperacillin/tazobactam, cefoperazone/sulbactam, cefazolin, cefuroxime, ceftriaxone, cefepime, ciprofloxacin, levofloxacin, moxifloxacin, amikacin, gentamicin, high level gentamicin, tobramycin, aztreonam, erythromycin, clindamycin, vancomycin, linezolid, tetracycline, trimethoprim/ sulfamethoxazole, and tigecycline.

2.4. Statistical Analyses. Data from the isolates and the susceptibility testing were analyzed using the WHONET 5.6 software provided by the World Health Organization. The Cochran-Armitage test was used to study the trends of the specimen numbers submitted from different outpatient clinics, different specimen types, and the antibiotic resistance percentages over time. This statistical analysis was performed using R 3.6.1 (R Foundation, Vienna, Austria) and a two-sided $P$-value 0.05 was considered as statistically significant.

\section{Results}

3.1. Specimens' Information. From 2013 to 2018, a total of 19,028 specimens were submitted to the clinical microbiology laboratory from 49 outpatient units. The total number of specimens increased from 2,303 in 2013 to 5,378 in 2018. As observed in Table 1, the number of specimens submitted from each department showed an increase over time. The top three units with the largest number of submissions were Emergency (30.7\%), Urology (18.9\%), and Nephrology (16.0\%). However, only the Emergency and Kidney Transplantation Clinic showed significant increases in the percentage of specimens submitted annually $(P<0.001)$.
Notably, the data from the Breast Surgery Clinic were incomplete and therefore were not included in the analysis. There were no significant differences for the percentages of specimen submission from the Peritoneal Dialysis Clinic. In contrast, all other departments showed a significant decrease in the annual percentage of specimen submission (Table 1).

3.2. The Different Types of Specimens Submitted for the Laboratory Tests of Microbiology. As shown in Table 2, the number of submitted specimens increased annually among different types of specimens, which was consistent with the increase of total number of specimen submission each year in Table 1. Sputum, whole blood, and pus showed a significant increase of specimen submission annually $(P<0.001)$. In contrast, urine, prostatic fluid, and other types of specimens showed a significant decrease of specimen submission annually $(P<0.001)$. The submissions of dialysate, peritoneal drainage, and pleural effusion and ascites had no significant changes over the period $(P>0.05)$.

3.3. The Microbial Growth between Different Types of Specimens. In a total of 19,028 clinical specimens, 4,719 (24.8\%) were tested positive for bacterial and fungal growth. The specimen types with high positive rates were peritoneal drainage $(67 \%(219 / 327))$, dialysate $(52.7 \%(267 / 507))$, pus (49.3\% (250/507)), and pleural effusion and ascites (31.9\% $(60 / 188))$, followed by urine $(28.9 \%(1,843 / 6,383))$, prostatic fluid $(28.1 \%(528 / 1,881))$, whole blood $(12.9 \%(405 / 3,139))$, and sputum $(9.7 \%(323 / 3,314))$.

3.4. Common Microbial Isolates and Species. In a total of 4,719 isolates of bacteria and fungi identified, after excluding duplicate isolates obtained from the same patient (each patient was sampled only once), there were 3,849 nonrepetitive isolates. These isolates belong to 211 different species, including 1,786 Gram-negative isolates (44.6\%), 1,744 Gram-positive isolates (45.3\%), 150 fungus isolates (3.9\%), and 169 isolates containing other species (4.4\%). The most frequently identified Gram-negative species were Escherichia coli, Klebsiella pneumonia and Pseudomonas 
TABLE 3: Annual number and percentage (\%) of bacterial isolates from the specimens between 2013 and 2018 from outpatients and patients visiting emergency department.

\begin{tabular}{|c|c|c|c|c|c|c|c|c|c|c|c|c|c|c|}
\hline \multirow{2}{*}{ Bacterial species } & \multicolumn{2}{|c|}{2013} & \multicolumn{2}{|c|}{2014} & \multicolumn{2}{|c|}{2015} & \multicolumn{2}{|c|}{2016} & \multicolumn{2}{|c|}{2017} & \multicolumn{2}{|c|}{2018} & \multirow{2}{*}{$P$ value } & \multirow{2}{*}{ Trend } \\
\hline & $N$ & $\%$ & $N$ & $\%$ & $N$ & $\%$ & $N$ & $\%$ & $N$ & $\%$ & $N$ & $\%$ & & \\
\hline \multicolumn{15}{|l|}{ Gram-negative } \\
\hline Escherichia coli & 134 & 70.2 & 149 & 66.8 & 128 & 61.0 & 138 & 53.5 & 218 & 60.2 & 268 & 56.9 & 0.471 & No change \\
\hline Klebsiella pneumonia & 21 & 11.0 & 24 & 10.8 & 14 & 6.7 & 29 & 11.2 & 38 & 10.5 & 56 & 11.9 & 0.400 & No change \\
\hline Pseudomonas aeruginosa & 9 & 4.7 & 19 & 8.5 & 11 & 5.2 & 18 & 7.0 & 18 & 5.0 & 32 & 6.8 & 0.760 & No change \\
\hline Enterobacter cloacae & 4 & 2.1 & 3 & 1.3 & 1 & 0.5 & 9 & 3.5 & 12 & 3.3 & 22 & 4.7 & 0.033 & $\uparrow$ \\
\hline Acinetobacter baumannii & 6 & 3.1 & 5 & 2.2 & 7 & 3.3 & 7 & 2.7 & 9 & 2.5 & 14 & 3.0 & 0.870 & No change \\
\hline Proteus mirabilis & 3 & 1.6 & 6 & 2.7 & 5 & 2.4 & 9 & 3.5 & 11 & 3.0 & 7 & 1.5 & 0.985 & No change \\
\hline Others* & 14 & 7.3 & 17 & 7.6 & 44 & 21.0 & 48 & 18.6 & 56 & 15.5 & 72 & 15.3 & & \\
\hline Total & 191 & 100.0 & 223 & 100.0 & 210 & 100.0 & 258 & 100.0 & 362 & 100.0 & 471 & 100.0 & & \\
\hline \multicolumn{15}{|l|}{ Gram-positive } \\
\hline Staphylococcus epidermidis & 37 & 18.1 & 81 & 28.1 & 37 & 20.4 & 29 & 12.7 & 45 & 18.0 & 39 & 11.3 & $<0.001$ & $\downarrow$ \\
\hline Staphylococcus aureus & 12 & 5.9 & 20 & 6.9 & 20 & 11.0 & 45 & 19.7 & 50 & 20.0 & 80 & 23.3 & 0.002 & $\uparrow$ \\
\hline Enterococcus faecalis & 21 & 10.3 & 33 & 11.5 & 20 & 11.0 & 30 & 13.2 & 33 & 13.2 & 56 & 16.3 & 0.984 & No change \\
\hline Staphylococcus haemolyticus & 34 & 16.7 & 39 & 13.5 & 22 & 12.2 & 12 & 5.3 & 11 & 4.4 & 20 & 5.8 & $<0.001$ & $\downarrow$ \\
\hline Streptococcus mitis & 5 & 2.5 & 16 & 5.6 & 16 & 8.8 & 17 & 7.5 & 20 & 8.0 & 28 & 8.1 & 0.556 & No change \\
\hline Enterococcus faecium & 9 & 4.4 & 7 & 2.4 & 10 & 5.5 & 17 & 7.5 & 19 & 7.6 & 32 & 9.3 & 0.163 & No change \\
\hline Others** & 86 & 42.2 & 92 & 31.9 & 56 & 30.9 & 78 & 34.2 & 72 & 28.8 & 89 & 25.9 & & \\
\hline Total & 204 & 100.0 & 288 & 100.0 & 181 & 100.0 & 228 & 100.0 & 250 & 100.0 & 344 & 100.0 & & \\
\hline
\end{tabular}

${ }^{*}$ Other gram-negative bacteria mainly include Enterobacteria, Pseudomonas, Proteus, Citrobacter, Haemophilus, Neisseria, Bacteroides, Aeromonas, and Morgan bacteria. ${ }^{* *}$ Other positive bacteria mainly include coagulase-negative Staphylococci, Streptococci, Enterococci, Micrococcus, Cooka, Lactobacillus, Corynebacterium, Actinomycete, Nocardia, and Clostridia.

TABLE 4: Antimicrobial resistance percentage (\%) of top four Gram-positive cocci among common antimicrobial agents between 2013 and 2018 from outpatients and patients visiting emergency department.

\begin{tabular}{|c|c|c|c|c|}
\hline Antimicrobial agent & $\begin{array}{l}\text { Staphylococcus aureus (\%) } \\
(n=227)^{*}\end{array}$ & $\begin{array}{c}\text { Staphylococcus epidermidis } \\
(\%)(n=268)^{*}\end{array}$ & $\begin{array}{l}\text { Enterococcus faecalis (\%) } \\
\qquad(n=193)^{*}\end{array}$ & $\begin{array}{l}\text { Enterococcus faecium } \\
(\%)(n=94)^{*}\end{array}$ \\
\hline \multicolumn{5}{|l|}{ Penicillins } \\
\hline Penicillin G & 100.0 & 96.6 & 0.0 & 94.7 \\
\hline Oxacillin & 25.1 & 74.6 & $* *$ & $* *$ \\
\hline Ampicillin & $* *$ & ** & 0.0 & 94.7 \\
\hline \multicolumn{5}{|l|}{ Fluoroquinolones } \\
\hline Ciprofloxacin & 17.6 & 32.1 & 23.8 & 96.8 \\
\hline Levofloxacin & 17.6 & 40.7 & 22.8 & 94.7 \\
\hline Moxifloxacin & 6.6 & 7.5 & $* *$ & $* *$ \\
\hline \multicolumn{5}{|l|}{ Aminoglycosides } \\
\hline Gentamicin & 23.3 & 8.6 & $* *$ & $* *$ \\
\hline Gentamicin HLAR & $* *$ & $* *$ & 0.0 & 0.0 \\
\hline \multicolumn{5}{|l|}{ Others } \\
\hline Erythromycin & 70.5 & 82.1 & 57 & 90.4 \\
\hline Clindamycin & 41.4 & 22 & $* *$ & $* *$ \\
\hline Vancomycin & 0.0 & 0.0 & 0.0 & 0.0 \\
\hline Linezolid & 0.0 & 0.0 & 0.0 & 0.0 \\
\hline Tetracycline & 41.4 & 20.1 & 66.8 & 45.7 \\
\hline $\begin{array}{l}\text { Trimethoprim/ } \\
\text { sulfamethoxazole }\end{array}$ & 29.5 & 64.6 & 0.0 & 0.0 \\
\hline Tigecycline & 0.0 & 0.0 & 0.0 & 0.0 \\
\hline
\end{tabular}

${ }^{*}$ The number of non-repetitive isolates for each species. ${ }^{* *}$ The result is not available.

aeruginosa, Enterobacter cloacae, Acinetobacter baumannii, and Proteus mirabilis. The most frequently identified Grampositive species were Staphylococcus epidermidis, Staphylococcus aureus, Enterococcus faecalis, Staphylococcus haemolyticus, Streptococcus mitis, and Enterococcus faecium. Table 3 shows the number and percentage (\%) of bacterial isolates from the outpatient specimens annually between
2013 and 2018. The percentage of $S$. aureus $(P=0.002)$ and E. cloacae $(P=0.033)$ demonstrated a significant increase annually, while $S$. epidermidis $(P<0.001)$ and S. haemolyticus $(P<0.001)$ showed a decrease. Candida albicans (78 isolates) and Aspergillus fumigatus (24 isolates) were the major fungi isolated, followed by Aspergillus flavus (9 isolates), Genus of Mucor and Fusarium (9 isolates), and 
TABLE 5: Annual multidrug resistance percentage ( $R \%)$ of bacterial isolates from the specimens between 2013 and 2018 from outpatients and patients visiting emergency department.

\begin{tabular}{|c|c|c|c|c|c|c|c|c|c|}
\hline Bacterial species & $\begin{array}{c}\text { Years } \\
\text { Multidrug resistance targets }\end{array}$ & $\begin{array}{c}2013 \\
R \% \\
\end{array}$ & $\begin{array}{c}2014 \\
R \% \\
\end{array}$ & $\begin{array}{c}2015 \\
R \% \\
\end{array}$ & $\begin{array}{c}2016 \\
R \% \\
\end{array}$ & $\begin{array}{c}2017 \\
R \% \\
\end{array}$ & $\begin{array}{c}2018 \\
R \% \\
\end{array}$ & $P$ value & Trend \\
\hline \multirow{2}{*}{ Escherichia coli } & ESBLs* & 62.6 & 59.6 & 57.4 & 59.3 & 56.8 & 59.4 & 0.586 & No change \\
\hline & $\mathrm{CRE}^{* *}$ & 0.0 & 0.0 & 0.0 & 0.0 & 0.0 & 0.0 & - & No change \\
\hline \multirow{2}{*}{ Klebsiella pneumoniae } & $E S B L s^{*}$ & 29.2 & 40.5 & 20.0 & 30.0 & 39.3 & 32.5 & 0.935 & No change \\
\hline & $\mathrm{CRE}^{* *}$ & 0.0 & 0.0 & 0.0 & 0.0 & 1.8 & 1.3 & 0.443 & No change \\
\hline Staphylococcus aureus & MRSA $^{\#}$ & 53.8 & 27.6 & 25.9 & 25 & 25 & 19.2 & 0.191 & No change \\
\hline Staphylococcus epidermidis & MRSE $^{\# \#}$ & 88.1 & 83.3 & 78.7 & 94.1 & 72.7 & 75.6 & 0.213 & No change \\
\hline
\end{tabular}

${ }^{*}$ ESBLs (extended spectrum $\beta$ lactamases): ESBLs multidrug resistance was evaluated by using ceftriaxone. ${ }^{* *} \mathrm{CRE}$ (carbapenem-resistant enterobacteriaceae): CRE multidrug resistance was evaluated by using imipenem and meropenem. ${ }^{\#}$ MRSA (methicillin-resistant S. aureus): MRSA multidrug resistance was evaluated by using oxacillin. ${ }^{\# \#}$ MRSE (methicillin-resistant S. epidermidis): MRSE multidrug resistance was evaluated by using oxacillin.

other Candida species (30 isolates). Other rare bacterial isolates were Corynebacterium (109 isolates), Lactobacillus (36 isolates), anaerobic bacteria (13 isolates) as well as Brucella, Eikenella, Actinomyces, Nocardia, and Nontuberculous Mycobacterium. Among Corynebacterium, Kroppenstedt Corynebacterium (23 isolates), and Ribbone Corynebacterium (10 isolates) were predominant.

\subsection{The Antimicrobial Resistance Profile of the Common Pathogens}

3.5.1. Staphylococcus spp. Our study indicated that all of $S$. aureus isolates in our community were resistant to penicillin (Table 4). However, many $S$. aureus strains, while resistant to penicillin, remained susceptible to penicillinase-stable penicillin, such as oxacillin. Strains resistant to oxacillin and methicillin were historically termed methicillin-resistant S. aureus (MRSA) [13]. Our study found that the percentage of MRSA isolates was $25.1 \%$ during the study period (Table 4) without significant changes annually (Table 5). In contrast, the CHINET reported that the prevalence of MRSA decreased from $69.0 \%$ in 2005 to $35.2 \%$ in 2017 [3]. The difference between our study and the CHINET is likely due to the lower prevalence of MRSA in community-acquired infections over the period in our study, suggesting the infiltration of MRSA isolates from hospitals to the community is very limited.

As shown in Table 4, less than $30 \%$ of S. aureus isolates were resistant to trimethoprim/sulfamethoxazole (29.5\%), gentamicin (23.3\%), ciprofloxacin (17.6\%), levofloxacin $(17.6 \%)$, and moxifloxacin (6.6\%), indicating a minor resistance to fluoroquinolones and aminoglycosides. In comparison, more than $30 \%$ of $S$. aureus isolates were resistant to erythromycin (70.5\%), clindamycin $(41.4 \%)$ and tetracycline $(41.4 \%)$. Like $S$. aureus, almost all of S. epidermidis isolates (96.6\%) in our community-acquired infections were resistant to penicillin (Table 4). Strains of S. epidermidis that were resistant to oxacillin and methicillin were historically termed methicillin-resistant S. epidermidis (MRSE). Our study showed that the percentage of MRSE isolates was $74.6 \%$ during the study period (Table 4 ) without significant changes annually (Table 5). Less than $30 \%$ of S. epidermidis isolates were resistant to clindamycin $(22.0 \%)$, tetracycline $(20.1 \%)$, gentamicin $(8.6 \%)$ and moxifloxacin
(7.5\%), whereas more than $30 \%$ of S. epidermidis isolates were resistant to erythromycin $(82.1 \%)$, compound sulfamethoxazole (64.6\%), levofloxacin (40.7\%), and ciprofloxacin (32.1\%). These findings indicate a moderate resistance of S. epidermidis to fluoroquinolones and a minor resistance to aminoglycosides. Significantly, all of S. epidermidis and S. aureus isolates collected from our study were sensitive to vancomycin, linezolid, and tigecycline.

3.5.2. Enterococcus spp. Among Enterococcus species, two major species (Enterococcus faecalis and Enterococcus faecium), were particularly human-specific pathogens. As indicated in Table 4, although the number of isolates of E. faecalis was significantly higher than that from E. faecium (193 isolates vs. 94), E. faecalis isolates were less resistant to most antibiotics compared to E. faecium isolates, including ampicillin ( $0.0 \%$ vs. $94.7 \%)$, erythromycin ( $57.0 \%$ vs. $90.4 \%)$, ciprofloxacin (23.8\% vs. $96.8 \%)$, penicillin (0.0\% vs. $94.7 \%)$, and levofloxacin (22.8\% vs. $94.7 \%)$; the only exception was tetracycline (66.8\% vs. $45.7 \%$ ). Furthermore, all of E. faecalis and E. faecium isolates collected from our study were sensitive to linezolid, tigecycline, and vancomycin.

3.5.3. Enterobacteriaceae. Enterobacteriaceae, including E. coli and $K$. pneumonia, produce extended-spectrum $\beta$-lactamases (ESBLs). ESBLs are a group of $\beta$-lactamases, which share the ability to hydrolyze $\beta$-lactam antibiotics, such as cephalosporins [14]. By using ceftriaxione as a substrate, our study suggested that $60.9 \%$ of $E$. coli isolates and $33.5 \%$ of $K$. pneumonia carried ESBLs (Table 6). Consistent with the ESBLs results, a similar trend was observed for the percentage of $E$. coli and $K$. pneumonia isolates resistant to other cephalosporins: cefepime (33.0\% vs. $5.9 \%)$, cefuroxime ( $80.7 \%$ vs. $23.5 \%)$, ceftazidime (54.5\% vs. $17.6 \%)$, and cefazolin (79.5\% vs. $17.6 \%)$ with the exception of cefotetan (5.7\% vs. $0.0 \%$ ) (Table 6). Less than $30 \%$ of E. coli isolates were resistant to tobramycin (21.6\%), cefoperazone/sulbactam (8.4\%), piperacillin/tazobactam $(8.0 \%)$, and amikacin $(2.3 \%)$. More than $30 \%$ of $E$. coli isolates were resistant to ampicillin (93.2\%), piperacillin (86.4\%), ciprofloxacin (80.7\%), levofloxacin (76.1\%), ampicillin/shubatan (70.5\%), trimethoprim/sulfamethoxazole (62.5\%), aztreonam (60.2\%), and gentamicin (39.8\%), indicating a high resistance to penicillins and fluoroquinolones. All of $E$. coli isolates were sensitive 
TABLE 6: Antimicrobial resistance percentage (\%) of top three Gram-negative bacilli among common antimicrobial agents between 2013 and 2018 from outpatients and patients visiting emergency department.

\begin{tabular}{|c|c|c|c|}
\hline Antimicrobial agent & Escherichia coli $(\%)(n=1035)^{*}$ & Klebsiella pneumoniae (\%) $(n=182)^{*}$ & $\begin{array}{l}\text { Pseudomonas aeruginosa (\%) } \\
\qquad(n=107)^{*}\end{array}$ \\
\hline \multicolumn{4}{|l|}{ Penicillins } \\
\hline Piperacillin & 86.4 & 17.6 & 14.9 \\
\hline Ampicillin & 93.2 & ${ }^{* *}$ & $-{ }^{* *}$ \\
\hline \multicolumn{4}{|l|}{ Cephems } \\
\hline Cefazolin & 79.5 & 17.6 & $-{ }^{* *}$ \\
\hline Cefuroxime & 80.7 & 23.5 & $-{ }^{* *}$ \\
\hline Ceftriaxone & 60.9 & 33.5 & $-{ }^{* *}$ \\
\hline Ceftazidime & 54.5 & 17.6 & 15.0 \\
\hline Cefepime & 33.0 & 5.9 & 7.5 \\
\hline Cefotetan & 5.7 & 0.0 & ${ }^{* *}$ \\
\hline \multicolumn{4}{|l|}{$\beta$-lactam combination agents } \\
\hline Ampicillin/Shubatan & 70.5 & 23.5 & ${ }^{* *}$ \\
\hline Piperacillin/Tazobactam & 8.0 & 0.0 & 7.5 \\
\hline Cefoperazone/sulbactam & 8.4 & 0.0 & 10.3 \\
\hline \multicolumn{4}{|l|}{ Fluoroquinolones } \\
\hline Ciprofloxacin & 80.7 & 11.8 & 0.0 \\
\hline Levofloxacin & 76.1 & 11.8 & 0.0 \\
\hline \multicolumn{4}{|l|}{ Aminoglycosides } \\
\hline Amikacin & 2.3 & 0.0 & 7.5 \\
\hline Gentamicin & 39.8 & 5.9 & 7.5 \\
\hline Tobramycin & 21.6 & 0.0 & 7.5 \\
\hline \multicolumn{4}{|l|}{ Others } \\
\hline Aztreonam & 60.2 & 5.9 & 22.4 \\
\hline Imipenem & 0.0 & 1.1 & 18.7 \\
\hline Meropenem & 0.0 & 1.1 & 18.7 \\
\hline Trimethoprim/sulfamethoxazole & 62.5 & 29.4 & ${ }_{-}^{* *}$ \\
\hline
\end{tabular}

${ }^{*}$ The number of non-repetitive isolates for each species. ${ }^{* *}$ The result is not available.

to imipenem and meropenem. As shown in Table 6, less than $30 \%$ of $K$. pneumonia isolates were resistant to compound sulfamethoxazole (29.4\%), ampicillin/shubatan (23.5\%), piperacillin (17.6\%), ciprofloxacin (11.8\%), levofloxacin (11.8\%), aztreonam (5.9\%), gentamicin (5.9\%), imipenem (1.1\%), and meropenem $(1.1 \%)$, indicating a minor resistance to these common antibiotics, such as penicillins and fluoroquinolones. All of the K. pneumonia isolates were sensitive to amikacin, piperacillin/tazobactam, cefoperazone/sulbactam, cefotetan, and tobramycin.

3.5.4. Nonfermentative Bacteria. All of $P$. aeruginosa isolates were sensitive to fluoroquinolones, including ciprofloxacin and levofloxacin. The $P$. aeruginosa-resistant isolates to other antibiotics were all less than $30 \%$ including aztreonam (22.4\%), metopenem (18.7\%), imipenem (18.7\%), ceftazidime $(15.0 \%)$, piperacillin (14.9\%), cefoperazone/sulbactam $(10.3 \%)$, amikacin $(7.5 \%)$, piperacillin/tazobactam $(7.5 \%)$, gentamicin $(7.5 \%)$, cefepime (7.5\%), and tobramycin $(7.5 \%)$, suggesting a minor resistance to these common antibiotics.

\section{Discussion}

In this study, we reported the microbial predominance and AMR profiling in a tertiary hospital using retrospective data from outpatient clinics and emergency department in a specific region of Northwestern China over six years. We will discuss our findings in the following five aspects.

4.1. The Medical Value to Study the Distribution of Specimen Submitted between Different Outpatient Clinics and Emergency Department. There are limited publications that compared the number and percentage of specimens submitted to clinical microbiology laboratories between different outpatient clinics. Different from other countries, patients in China can directly visit healthcare services without referral requirements from primary care doctors. Our study showed a trend of increasing number of specimen submissions in each clinic (Table 1), likely associated with the increased population in the communities. The departments of Emergency, Urology, and Nephrology were among the top three clinics that submitted specimens for microbiological studies. Urinary tract infection has been considered the major reason for outpatients to visit the Urology and Nephrology departments [15] and is also one of the major concerns for the Emergency visits [16]. More importantly, our study revealed that only two departments, the Emergency Department and Kidney Transplantation Clinic, demonstrated a significant increase of specimen submitted annually $(P<0.001)$. This indicates that these two departments, particularly the Emergency Department 
[17], are in the frontline of infection control. The increased importance of the Emergency Department is likely associated with enhanced medical functions of the emergency service. There is a trend for the Emergency Department to handle more previously inpatient cares. The increased importance of the Kidney Transplantation Department indicates that Kidney Transplantation is becoming a mainstream medical service and repeated infection after kidney transplantation is an urgent medical issue in outpatient clinics [18].

4.2. Relatively Lower Ratio of Gram-Negative Isolates in Our Community-Acquired Infections. Among 3,849 nonrepetitive isolates, the ratio of Gram-negative to Grampositive was nearly $1: 1$ ( $45.3 \%$ vs. $46.4 \%)$ in our study while the ratio reported by the CHINET was roughly $2: 1$ with approximately $70 \%$ of Gram-negative bacteria $[3,4]$. This difference is probably because that the CHINET data were significantly derived from inpatient service and our data were from a mixture of outpatient and inpatient services. Studies from US hospitals reported that Gram-negative bacteria were more common in cases with ventilator-associated pneumonia and urinary tract infections [19] and Gram-negative bacteria were the dominant type of infection at the intensive care units [20]. These results suggest that there is a likely association between the higher ratio of Gram-negative infections and the inpatients experiencing invasive medical devices and surgical procedures. Indeed, the Gram-negative bacteria contain highly efficient mechanisms of antibiotic drug resistance [21]. By comparing Tables 4 and 6 in this study, Gram-negative bacteria, particularly E. coli, appeared more resistant to the antibiotics and required more complicated therapeutic regimens. Overall, relatively lower ratio of Gram-negative isolates was likely a general feature in outpatient service.

\subsection{Vancomycin, Linezolid, and Tigecycline Were the Most} Effective Antibiotics for Patients with Gram-Positive Infection in Our Community. Vancomycin, linezolid, and tigecycline have been used to treat multidrug resistant bacteria in the community [22]. In our study, Staphylococci and Enterococci (Table 4) were $100 \%$ sensitive to vancomycin and linezolid, which was in general consistent with the findings from the CHINET. For example, the CHINET surveillance in 2018 showed that Staphylococci isolated from blood, urine, the lower respiratory tract, and cerebrospinal fluid were 100\% sensitive to vancomycin and linezolid except less than $1 \%$ of Staphylococci isolated from blood was resistant to linezolid [4]. Moreover, less than $5 \%$ of E. faecium and less than $1 \%$ of E. faecalis were vancomycin-resistant in the CHINET surveillance from 2005 to 2017 [3]. These findings indicate that vancomycin and linezolid remain the most effective treatment for Gram-positive infection for both hospital and community acquired infections.

In our study, Staphylococci and Enterococci (Table 4) were also $100 \%$ sensitive to tigecycline, which was an expanded broad-spectrum intravenous glycylcycline antibiotic. This finding suggests that tigecycline is a primary antibiotic treatment for multidrug-resistant bacterial infection in our communities.

4.4. Carbapenems Were the Most Effective Treatment for Patients with Gram-Negative Infections in Our Communities. Table 6 shows higher resistance rates of Gram-negative pathogens, particularly E. coli, to the cephalosporins and quinolones in this study. To overcome the clinical challenge, carbapenems such as imipenem and meropenem have been used for these multidrug-resistant bacterial infections [23]. Table 6 also shows that the percentages of E. coli, $K$. pneumonia and $P$. aeruginosa isolates resistant to imipenem were $0.0 \%, 1.1 \%$, and $18.7 \%$, respectively. Moreover, the annual rate of resistance of E. coli and K. pneumonia to carbapenems showed no significant increase as shown in Table 5. These findings support that carbapenems should be a primary antibiotic for multidrug-resistance bacterial infections in our communities.

\subsection{There Was No Significant Increase in Multidrug-Resistant} Bacteria Observed in Our Communities. Tables 4 and 6 show higher resistance rates between certain bacteria and antibiotics, such as methicillin-resistant Grampositive pathogens (MRSA and MRSE) and extended spectrum $\beta$ lactamases (ESBLs)-resistant Gram-negative pathogens. By using ceftriaxone and oxacillin, Table 5 indicates an annual change of the resistance rate of multidrug-resistant bacteria in recent years. A significant increase of resistance rate would require immediate intervention to investigate the reason. The results in Table 5 suggest that there is no significant increase of multidrug-resistant bacteria, indicating a reasonable prescription of antibiotics in our outpatient and emergency services and successful physician education on the prevention of antibiotic resistance. The main limitation in this study was that this was a single-center retrospective observational study. It should be cautious to translate our results to other hospitals. However, the quality of clinical sampling procedures and techniques were in general better controlled in a single center than multiple centers. First, they also allowed us to obtain a representative specimen in many clinical departments throughout the hospital. When we repeated the sample collection and analysis annually, we could observe trends over a period of time. In addition, a single-center study, when appropriately performed, is a useful and inexpensive surveilling tool to reflect the regional situation of prevailing microorganisms and their resistance to antimicrobials. Second, outpatient surveillance is an important tool to study community-acquired infections. It should be emphasized that not all outpatient and inpatients visiting the Emergency Department with infections are contracted from the communities. This is the reason we only used the term of "community settings" in this study since the patients seeking medical care in our outpatient and emergency services come directly from the community. 


\section{Data Availability}

All the data are available upon request.

\section{Ethical Approval}

This project was approved by the Institutional Review Board of the First Affiliated Hospital of Xi'an Jiaotong University (No: XJTU1AF2017LSK-83).

\section{Consent}

Written informed consents cannot be obtained because the laboratory tests for microbiology are part of standard care and the records of the patient's personal identities were removed prior to data analysis.

\section{Conflicts of Interest}

There are no conflicts of interest.

\section{Authors' Contributions}

Caifeng Wang was responsible for study design, funding application, and manuscript writing and managed the study. Jine Lei performed laboratory analysis. Wen Li and Yali Li performed data entry. Juanjuan Gao performed statistical analysis of data. Dancheng Zhang and Fang Li performed data analysis.

\section{Acknowledgments}

The authors would like to acknowledge the support provided by the Shaanxi Key Science and Technology Project, China, for funding this work (To JL, grantnumbers: 2017SF-198).

\section{References}

[1] L. S. J. Roope, R. D. Smith, K. B. Pouwels et al., "The challenge of antimicrobial resistance: what economics can contribute," Science, vol. 364, no. 6435, Article ID eaau4679, 2019.

[2] G. Croxall, J. Hale, V. Weston et al., "Molecular epidemiology of extraintestinal pathogenic Escherichia coli isolates from a regional cohort of elderly patients highlights the prevalence of ST131 strains with increased antimicrobial resistance in both community and hospital care settings," Journal of Antimicrobial Chemotherapy, vol. 66, no. 11, pp. 2501-2508, 2011.

[3] F. Hu, D. Zhu, F. Wang, and M. Wang, "Current status and trends of antibacterial resistance in China," Clinical Infectious Diseases, vol. 67, no. suppl_2, pp. S128-S134, 2018.

[4] F. Hu, Y. Guo, Y. Yang et al., "Resistance reported from China antimicrobial surveillance network (CHINET) in 2018," European Journal of Clinical Microbiology \& Infectious Diseases, vol. 38, no. 12, pp. 2275-2281, 2019.

[5] F. P. Hu, Y. Guo, D. M. Zhu et al., "Resistance trends among clinical isolates in China reported from CHINET surveillance of bacterial resistance," Clinical Microbiology and Infection, vol. 22, no. Suppl 1, pp. S9-S14, 2016.

[6] D. Yin, S. Wu, Y. Yang et al., "Results from the China antimicrobial surveillance network (CHINET) in 2017 of the in vitro activities of ceftazidime-avibactam and ceftolozanetazobactam against clinical isolates of enterobacteriaceae and
Pseudomonas aeruginosa," Antimicrobial Agents and Chemotherapy, vol. 63, no. 4, Article ID e02431-18, 2019.

[7] Y. Xiao, Z. Wei, P. Shen et al., "Bacterial-resistance among outpatients of county hospitals in China: significant geographic distinctions and minor differences between central cities," Microbes and Infection, vol. 48, no. 6, pp. 417-425, 2015.

[8] World Health Organization, Antimicrobial Resistance: Global Report on Surveillance, World Health Organization, Geneva, Switzerland, 2014.

[9] S. Li, Y. Guo, C. Zhao et al., "In vitro activities of tedizolid compared with other antibiotics against gram-positive pathogens associated with hospital-acquired pneumonia, skin and soft tissue infection and bloodstream infection collected from 26 hospitals in China," Journal of Medical Microbiology, vol. 65, no. 10, pp. 1215-1224, 2016.

[10] L. Han, J.-E. Lei, X. Wang et al., "Septicemia caused by leifsonia aquatica in a healthy patient after retinal reattachment surgery," Journal of Clinical Microbiology, vol. 51, no. 11, pp. 3886-3888, 2013.

[11] C. Zhao, F. Zhang, Y. Chu et al., "Phenotypic and genotypic characteristic of invasive pneumococcal isolates from both children and adult patients from a multicenter surveillance in China 2005-2011," PLoS One, vol. 8, no. 12, Article ID e82361, 2013.

[12] J.-E. Lei, S. Han, W. Wu, X. Wang, J. Xu, and L. Han, "Extensively drug-resistant Acinetobacter baumannii outbreak cross-transmitted in an intensive care unit and respiratory intensive care unit," American Journal of Infection Control, vol. 44, no. 11, pp. 1280-1284, 2016.

[13] https://gram.cdc.gov/hai/settings/lab/lab_mrsa.html.

[14] T. Dimitriu, F. Medaney, E. Amanatidou, J. Forsyth, R. J. Ellis, and B. Raymond, "Negative frequency dependent selection on plasmid carriage and low fitness costs maintain extended spectrum $\beta$-lactamases in Escherichia coli," Scientific Reports, vol. 9, no. 1, p. 17211, 2019.

[15] D. C. Fiore and C. L. Fox, "Urology and nephrology update: recurrent urinary tract infection," FP Essentials, vol. 416, pp. 30-37, 2014.

[16] S. B. Dubbs and S. K. Sommerkamp, "Evaluation and management of urinary tract infection in the emergency department," Emergency Medicine Clinics of North America, vol. 37, no. 4, pp. 707-723, 2019.

[17] R. D. Mistry, P. S. Dayan, and N. Kuppermann, "The battle against antimicrobial resistance: time for the emergency department to join the fight," JAMA Pediatrics, vol. 169, no. 5, pp. 421-422, 2015.

[18] Y.-X. Chen, R. Li, L. Gu, K.-Y. Xu, Y.-Z. Liu, and R.-W. Zhang, "Risk factors and etiology of repeat infection in kidney transplant recipients," Medicine, vol. 98, no. 38, Article ID e17312, 2019.

[19] A. I. Hidron, J. R. Edwards, J. Patel et al., "NHSN annual update: antimicrobial-resistant pathogens associated with healthcare-associated infections: annual summary of data reported to the national healthcare safety network at the centers for disease control and prevention, 2006-2007," Infection Control \& Hospital Epidemiology, vol. 29, no. 11, pp. 996-1011, 2015.

[20] R. A. Weinstein, R. Gaynes, J. R. Edwards, and N. N. I. S. System, "Overview of nosocomial infections caused by gram-negative bacilli," Clinical Infectious Diseases, vol. 41, no. 6, pp. 848-854, 2005. 
[21] A. Y. Peleg and D. C. Hooper, "Hospital-acquired infections due to gram-negative bacteria," New England Journal of Medicine, vol. 362, no. 19, pp. 1804-1813, 2010.

[22] D. Van Duin and D. L. Paterson, "Multidrug-resistant bacteria in the community: trends and lessons learned," Infectious Disease Clinics of North America, vol. 30, no. 2, pp. 377-390, 2016.

[23] P. M. Hawkey and D. M. Livermore, "Carbapenem antibiotics for serious infections," BMJ: British Medical Journal, vol. 344, Article ID e3236, 2012. 